Ann. Abeille, I964, $7(4), 329-347$.

\title{
ÉTUDE DU SPECTRE POLLINIQUE DE QUELQUES MIELS ESPAGNOLS
}

\author{
T. LOLTEAUX et Ph. VERGLRON \\ Station de Recherches sur l'Abeille et les Insectes soitaux, \\ Bures-su-Tette (Seine-el-Oise)
}

SOMMAIRE

Les aluteurs ont sćlectiomné 38 miels espagnols et en ont dressé le spectre pollinique. Quelques familles ont été choisies : Papavertcées, ('istacées, Éricacées et Labiées. L'étude de l'aire de répartition des principales plantes représentantes de ces familles et de la morphologie des grains de pollen correspondants a permis de dégager des espèces caractéristiques des spectres des miels espagnols.

\section{IN'TRODUC'TION}

Depuis quelques années, de nouveaux courants commerciaux ont anené sur le marché français des tonnages importants de miels espagnols ( 865 tonnes en I960, 285 I tonnes en I06I, I 283 tonnes en 1062, 2 I87 tonnes en I063) dans les qualités les plus diverses. I es cours de ces miels étant en général nettennent inférieurs à cenx des miels français similaires, la différence de prix a été l'origine d'un certain nombre de fraudes sur les appellations qu'il importait de pouvoir détecter rapidement par l'analyse pollinique.

Dès d'abord, la recherche de l'origine géographique das miels suspects nous a posé des problèmes relativenent délicats car on ne possédait, il y a encore quelques années, que des connaissances très sommaires sur les miels espagnols. On avait bien remarqué leur richesse en espèces mais très peu d'entre elles avaient pu être déterninées avec toute la précision désirable. I a bibliographie était rare puisqu'on ne pouvait guère se reporter qu'au travail de VIEI'LZ (I $950-105 \mathrm{I}$ ) s11 les miels de Galice 
qui fournit certes de précieux renseignements mais ne concerne qu'une faible partie du territoire espagnol. Les analyses effectuées par MItrrizio (I938-I955) n'apportaient que des éléments d'information fragmentaires car cet auteur n'avait pas abordé le problème des miels espagnols de façon directe. Un mémoire de MARTixs d'AI'TE (I95I), fort intéressant par certains cótés, ne concernait cependant que les miels portugais ; à ce titre, il constituait une contribution à l'étude des miels de la péninsule ibérique mais d'une portée très limitée.

Nous nous trouvions donc, pour pouroir être en mesure de reconnaitre à coup sûr les miels importés d'Espagne, dans la nécessité d'en entreprendre une étude approfondie en utilisant le riche matériel mis à notre disposition par 1'intensification des importations. C'est le résultat de ces investigations poursuivies pendant plusieurs années que nous exposons ici.

\section{MATÉRIEI, E'I MÉTHODES}

Les préparations de pollens de rélérence ont été obtenues par la méthode habituelle (Matrizio et louveaux, 1963$)$. Toutefois, pour certaines espices. num avoms fait appel aux collections du Laboratoire de Palyonologie du Muséun national d'Histoire naturelle. Il s'agissait dans ce cas de matériel fossilisé.

lets préparations de niels ont été réalisées. elle aussi. selon lat méthode classique panurzio et Louveaux, ig63). Seule modification notable apportée à cette méthode, l'utilisation d'acide sulfurique N/ro pour diluer le miel avant lit centrifugation: une seconde cent rifugation dans l'eau distillée élimine l'acide avaut le montage du culot. Cette méthode permet souvent d'obtenir des préparations plus claires par élimination de produits minéraux insolubles dans l'eau pure tels que l'oxalate de calcimm par excmple.

Pour chaque miel étudié, nous arous procédé. sous le microseope, à un inventaire aussi complet que possible des formes présentes. Nous 1 lavons pas effectué de numérations, ceci pour les raisons qui sont exposées par l'un d'entre nous dans une autre communication présentée à ce colloque (VERGRRON, 1964). Cependant, afin de met tre en éridence les especes les plus importantes et celles qui n'interviennent que de façon accessoire, mus avons distingué, selon la nomenclat ure habituelle, des pollens "dominants", " "laccompagnement" nu "isrlés", ces termes correspondant aux trois grandes classes traditionnelles de fréfuence. Pour opérer ce classentent, nous avons tenu compte des connaissances actuelles sur la richése spéciticue en pollen des différents miels unifloraux. Il en résulte que, dans ce travail, nous pourons très bien présenter Rosmarinus comme dominant, par exemple, même si numériguement il e,t dominé par le pollen d'une plante telle que Castanea. Bien que cette méthode fasse appel à une estination basée sur lexpérience et sur les donnés bibliographiques, elle about it à une représentation rles faits plus proche de la réalité que les numérations qui n'apportent souvent qiume illustire précioion.

Par ailleurs, nous devons signaler que nows avons procele parfois à cles regroupements d'espèces et traité comme une "association" lau sons des phytogéographes) un ensemble de pollens cohérent, représentat if d'un milieu végétal déterniné. C'st ainsi par exemple que le couple Kosmarinus - Helianthemum est traité comme un tout caractérisant patrfaitement les miels de Romarin Dans ce cas, les différents pollens de lassuciation sont groupés tans la même classe de fréquence. Cette remarape permet de comprendre pourquoi dans le tableau s. un mîme niel peut présenter plusieurs pollens "dominants", ce qui est contraire à lat règle traditionnelle (ZANDER 1935, 194 I ; Matrizio 1949). Nous avons tenté, par cette méthode, de nous rapprocher des technigues des phytogéographes pour lesquels la "dominante "d'une atsociation nest pas toujours la plante numériquement la plus inportante d'un relevé mais celle yui lui donne son caractère propre.

Les 38 miels espagnols qui font lobjet de conte étude ont été sélectionnés parmi de très nombreux autres parce quils représentaient des types courants et qu ils pouvaient généralentent recevoir une appellation florale bien déteminée. D'autre part, nous avons choisi uniquentent les produits présentant loute garantic diauthenticité du point de vue de lorigine géographique. Enfinnous avons jugé préférable de nous limiter it un nombre restreint d'échintillons pour éviter d"inutiles répétitions. En effet beaucoup de spectres se retruvent avec une grande similitude d'un échantillon a lautre. 


\section{ÉLÉMENTS ESSENTIEIS DU SPECTRE POILINIQUE}

\section{DES MIELS ESPAGNOLS}

Dès qu'on aborde l'étude des miels espagnols, on est frappé par la richesse en espèces des spectres obtenus. On constate rapidement la dominance de quelques familles telles que les Éricacées, les Cistacées, les Boraginacées, les Papilionacées, les Labiées et la présence fréquente des genres Citrus, Olea, Eucalyptus, etc. Bien souvent, le caractère espagnol d'un miel apparaît clairement, du fait même qu'on note la présence de combinaisons de pollens qui ne sauraient se rencontrer facilement ensemble ailleurs que dans la péninsule ibérique.

Nous avons regroupé dans le tableau I les spectres polliniques des 38 miels étudiés. Pour plus de commodité, notus avons groupé les échantillons sous les appellations habituelles des miels, c'est-à-dire en nous référant à l'espèce végétale dominante. Nous avons ainsi distingué des miels de Lavande, de Thym, d'Oranger, de Romarin, de Bruyère, de Vipérine, de Ronce, de Sainfoin et, enfin, des miels ne présentant pas de dominance nette, sans propriétés physico-chimiques ou organoleptiques bien caractéristiques et que nous appelons "miels toutes fleurs". Examinons plus en détail les spectres obtenus.

\section{Io Miers DE LAVANDE (Lavandula).}

Alors que les miels de Lavande français constituent un ensemble relativement homogène, tant du point de vue pollinique qu'organoleptique et physico-chimique les miels de Lavande espagnols sont très variés. Les miels de Lavandula vera existent en Espagne. Leur spectre pollinique comporte un nombre d'espèces bien plus grand que leurs homologues français : en moyenne 25 espèces. Lavandula vera y est associée d'une façon très générale à d'autre Labiées et, en particulier à Lavandula stoechas (Miel no 696). Cette association n'existe pas en France, sauf parfois dans certaines régions des Pyrénées orientales; mais les miels espagnols présentent dans leur spectre, en même temps que L. stoechas, Hypecoum. Le couple Lavandula stoechas-Hypecoum est très fréquent dans les spectres de miels espagnols (tabl. 2). De plus, tous les miels de Lavande d'Espagne présentent dans leur spectre une grande variété de Cistacées, parfois en pourcentage élevé, ce qui n'est pas carac. téristique des miels français. Les miels de Lavandin n’ont pas été tronvés en Espagne.

\section{$2^{\circ}$ Mress DE THYM (Thymus)}

Les miels de Thym sont nettement moins variés que les précédents. La richesse en Cistacées constitue le seul point commun. Le nombre des espèces est plus faible, restant en général autour de la vingtaine. L'association Thymus, Hypecoum, Arbres fruitiers, Papilionacées type Ulex est très constante.

\section{$3^{\circ}$ MiELS D'ORANGER (Citrus)}

Les miels d'Oranger n'existent pas en France. I es miels espagnols de ce type sont peu variés. Leur spectre pollinique est pauvre en espèces (en général une quinzaine) et les miels eux-mêmes sont souvent pauvres en pollen (Maurizio, I958). 
I'Olivier (Olea), les Arbres fruitiers et surtout l'Amandier (Amygdalus) constituent avec Citrus la base du spectre pollinique. Il convient de noter qu'Olea peut atteindre des pourcentages élevés, ce qui est très rare en France. Enfin, signalons que les miels d'Oranger sont les seuls miels espagnols à présenter un spectre pollinique pauvre en Cistacées.

\section{$4^{o}$ Miels de romarin (Rosmarinus)}

Les miels de Romarin espagnols constituent un ensemble bien homogène, très différent de ce qu'on observe dans les miels français de même origine florale. La base du spectre comporte très régulièrement, à côté de Rosmarinus, Hypecoum, Helianthemum, Cistus crispiss, Amygdalus, Arbres fruitiers et Papilionacées type Ulex.

\section{$5^{\circ}$ MiELS DE BRUyìre (Éricacées)}

Les miels de Bruyère sont, au contraire des précédents, très variés. Les produits du nord-ouest de l'Espagne et ceux des régions plus mériđionales sont très différents les uns des autres mais, en aucun cas, ils ne se rapprochent des miels de Bruyère français. Les miels de Callune (Calluna) existent en Espagne mais le pollen de cette plante y est le plus souvent associé à celui d'Erica vagans qui se retrouve dans tous les miels de la moitié occidentale de 1'Espagne. Les miels français de Callune produits dans le Sud-Onest ont un spectre très particulier (Maurizio et LouvEaux, I964), qu'on ne retrouve pas en Espagne.

Les miels d'Erica umbellata ( $\mathrm{n}^{0} 3 \mathrm{I} 2$ et 686 ) n'existent pas en France. I1s ont un spectre pollinique complexe, riche en Cistes et en Lavandes: Cistus ladaniferus, Cistus heterophyllus, Lavandula stoechas, Lavandula dentata.

\section{MIEI,S DE VIPÉRINE (Echium)}

Les miels de Vipérine sont très rares en France mais, par contre, courants en Espagne. Les spectres polliniques des miels où domine Echium comportent un grand nombre d'espèces. On y trouve, accompagnant la Vipérine, Evica umbellata, un grand nombre de Cistacées et des Labiées (Lavandula stoechas et Lavandula dentata).

\section{$7^{\circ}$ MIEISS DIVERS}

A côté de tous les miels qui viennent d'être décrits et qui présentent une caractéristique florale nette, on trouve parmi les produits espagnols des miels variés plus ou moins typiques. L'échantillon $\mathrm{n}^{0} 725$ par exemple présente Onobrychis sativa comme pollen dominant. Il ne s'agit pas d'un produit exceptionnel car les miels de sainfoin sont communs en Espagne ; ils se distinguent parfaitement des miels français de même or igine florale par leur caractère essentiellement méditerranéen ; leur spectre polliniqge comporte des Cistacées et des Labiées ainsi qu'Hypecoum.

Le spectre pollinique du miel no Io est assez semblable à ceux qui ont été décrits par VIEITEZ (I950) en Galice comme miels de Ronce (Rubus).

Les miels " toutes fleurs " restent toujours caractérisés par la complexité du spectre et la richesse très générale en Cistacées. 


\section{DISCUSSION}

L'examen du tableau I montre que nous avons observé dans les miels espagnols un certain nombre d'espèces qui n'ont jamais été décrites dans les miels. Ainsi, à notre connaissance, la présence du pollen d'Hypecoum dans les miels n'avait jamais été signalée; en ce qui concerne les Cistacées, on s'était borné jusqu'ici à noter la présence du genre Cistus sans aboutir à l'espèce.

Il importe donc de discuter maintenant:

I $^{\circ}$ L'intérêt de certains pollens pour l'identification des miels espagnols.

$2^{\circ}$ I a possibilité de déterminer avec précision les pollens en question. Entin, il nous paraît nécessaire d'aborder le problème de la richesse en espèces des miels d'Espagne.

\section{Io lees espéces caractérisliques}

Devant la très grande richesse en espèces des miels d'ispagne, nous avons dû renoncer à identifier avec précision tout ce qui se présentait sous l'objectif du microscope. Nous avons préféré nous liniter à un certain nombre de familles ou de genres qui nous ont paru susceptibles de nous fournir les espèces les plus intéressantes du point de rue de la caractérisation des miels espagnols. Pour l'étude de la répartition géographique, nous avons utilisé les différentes flores disponibles, tant de France

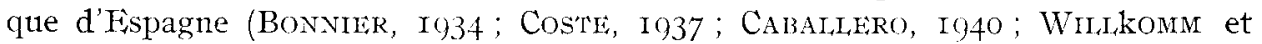
LANGi:, I870).

PAPAVíRAcḱss. -- La famille des Papaveracées nous a fourni un élément particulièrement intéressant avec le genre Hypecoum. Fin France, Hypecoum procumbens se rencontre dans les champs calcaires ensoleillés du Midi et dans la basse vallée du Rhône ; c'est une plantı relativement rare. Par contre, elle est commune en Espagne oì le genre tout entier est abondamment représenté.

Le pollen d'Hypcoum procumbens est extrêmement caractéristique et ne peut être confondu avec aucun autre (HowTMx, I952). Il est dicolpé, presque sphérique ou légèrement prolate $(20 \times$ Is $\mu$ ). Son exine est mince. I) ans les miels comne dans les préparations de référence, sa coloration jaune intense retient l'attention.

I.e pollen d'Hyccoum, qu'il s'agisse de l'espèce procumbens ou d'espèces voisines, se retrouve dans 24 des 38 spectres pollini(utes de niels espagnols étudiés. On peut donc considérer que c'est un élément fréquent dans les miels d'Espagne. Il peut se rencontrer dans toutes les classes de fréquence et même comme "dominant " dans certains cas. Par contre, dans les miels français, on peut considérer qu'il est toujours absent bien que théoriquement :a préşilce ne soit pas totalement inpossible. Sur l'ensemble des miels français que nous avons examinés jusqu'ici, soit près de 2 ooo, nous ne l'avons trouvé clu'une seule fois et à l'état isolé ; il s'agissait d'un miel de la Drôme qui n'avait par ailleurs aucun caractère espagnol alors que dans les miels de la péninsule ibérique Hypecoum se trouve toujours associé à d'autres éléments caractéristicues.

ÉrRICACÉEs. - I es miels espagnols sont souvent très riches en Firicacées. Il importait 
donc de saroir si certaines d'entre elles n'étaient pas susceptibles de constituer d'intéressantes caractéristiques. Parmi celles qui présentent un intérêt apicole suffisant nous en avons retenu et étudié du point de vue de leur répartition géographique un certain nombre (fig. 2). Yoyons ce qu'on peut tirer de cette étude.

Genre Callunta. Calluna vulgaris se trouve dans les régions siliceuses de presque



FIG. 2. - A. Limiti orientale el méridionale de laive de réfartition des bruyères "océaniques" (Erica ciliaris, Irica tetralix, Erica cinerea)

13. Limite orientale el mérilionale de l'aire de répartition de Erica vagans.

C. Limite septentrionale de laive de répartition de lirica arborea.

D. Limite seftentrionale de l'aive de répartition de Lisia umbellata.

toute l'Europe. Elle se rencontre dans toute la France et dans toute l'Ispagne sur terrains siliceux, aussi bien en plaine cu'en altitude.

Genre Arbutus. Arbutus unedo existe en Europe méridionale et occidentale; on le trouve jusqu'en Islande. Il est présent aussi bien en France qu'en Esspagne, surtout dans les régions côtières.

Genre Taccinium. Taccinium myrtillus se rencontre dans les régions montagneuses, aussi bien en Esspagne qu'en I'rance.

Genre Rhododendron. Rhodendron ferrugineum se rencontre dans les régions montagneuses aussi bien en Iispagne qu'en France.

Genre Erica. Ce genre comporte de nombrenses espèces risitées par les abeilles:

- Erica carnca. Présente en France dans les régions alpestres et surtout en 
Savoie et Haute-Savoie. Signalée dans les Alpes-Maritimes. N'existe pas en Eispagne. - Evica tetralix, Erica cinerea, Erica ciliaris. On peut grouper ces trois espèces dont l'aire géographique est assez semblable. Il s'agit de plantes surtout océaniques qui peuvent se rencontrer depuis le nord et le nord-ouest de la France jusqu'au Portugal. Elles sont courantes en Espagne: Galice, Leon, Asturies, Cantabrique, Navarre, Aragon, les deux Castilles.

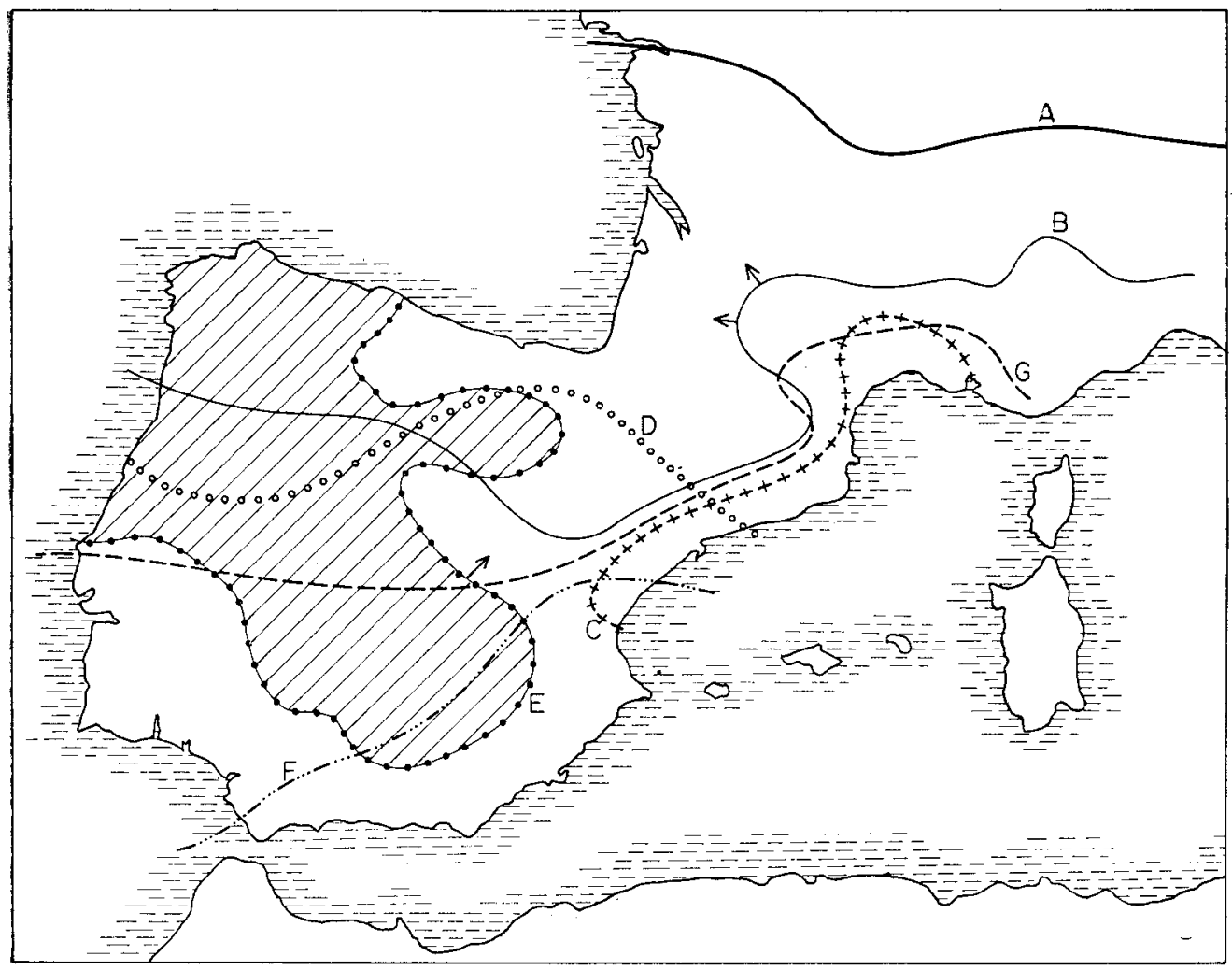

FIG. 3. - A. Limite septentrionale de l'aire de répartition de Cistus salviaefolius.

B. Linite septentrionale de l'aire de répartition de Cistus crispus, Cistus albidus, C. monspeliensis, C. laurifolius.

C. I imite de l'aive de répartition de Cistus varius.

D. Limite seplentrionale de l'aire de Cistus heterophyllus.

I. Limite de laire de Cistus hirsutus.

F. Limite septentrionale de l'aire de Cistus mumbyi et Cistus libanotis.

G. Limite septentrionale de l'aire de Cistus ladaniferus.

- Erica vagans. Courante dans le sud-ouest de la France, plus rare dans le centre et la région parisienne, elle a en Espagne la même répartition que les trois espèces précédentes.

- Erica arborea. Commune dans la région méditerranéenne, aussi bien en France qu'en Espagne.

- Erica umbellata. Cette espèce n'existe pas en France; par contre, elle est abondante en Espagne (VIEITEz, I950) boréale et austro-occidentale (Asturies, Galice, Leon, les deux Castille, Estremadure, régions orientales, Grenade). 
L'intérêt d'Evica umbellata en tant que caractéristique des miels espagnolsapparaît donc nettement. C'est la seule Bruyère qui soit inconnue en France et abondante en Espagne où elle constitue par endroits une ressource mellifère importante. $E$. umbellata se retrouve dans I4 relevés sur 38 (tab1. I).

Le pollen d'Erica umbellata est bien caractéristique. Il a été décrit par J. A. MarTIN d'ALTE (I95I) et par OLDFIEID (I959). Les grains de la tétrade sont très arrondis et bien individualisés; c'est un des pollens d'Ericacées qui a le plus grand rapport $\mathrm{D} / d(\mathrm{D}=35$ à $50 \mu, \quad d=23$ à $30 \mu, \quad$ Moyenne $\mathrm{D}=42 \mu, \quad d=26 \mu)$. L'exine est assez épaisse, peu ornementée ; les sillons sontlongs et étroits.

Avant de quitter les Éricacées signalons que les miels présentant Erica vagans comme pollen dominant sont beaucoup plus fréquents en Espagne qu'en France. Lorsqu' on se trouve devant les miels présentant l'association Calluna vulgaris-Erica vagans, on constate que les pollens d'accompagnement ne sont pas les mêmes en France et en Espagne ; en France (Sud-Ouest) l'association normale comporte Jasione montana, Ulex, Evica cinerea, Rhamnus frangula, Castanea sativa; en Espagne ce sont au contraire les Cistacées et les Labiées qui dominent.

Cistacées. - Les Cistacées constituent sans doute la famille la mieux représenćée dans les miels d'Espagne. En nous limitant au genre Cistus, nous avons procédé à une étude des espèces les plus intéressantes (fig. 3). Du point de vue de la répartition géographique, on peut dresser le tableau suivant:

- Genve Cistus. Cistus albidus, C. monspeliensis, C. crispus, C. salviaefolius, C. laurifolins sont communs à la France et à l'Espagne et on trouve leur pollen dans les miels des deux pays.

- Cistus varius. Présent dans le Gard, l'Aveyron et la région de Barcelone. Espèce peu importante, sauf en Afrique du Nord.

- Cistus libanotis et C. mumbyi. Ces deux espèces sont particulières à 1'Espagne du sud et à toute l'Afrique du Nord.

- Cistus ladaniferus. Présent dans toute 1'Espagne, abondant dans les régions centrale et austro-occidentale. Assez courant en France dans 1'Aude et les Pyrénées orientales; signalé par certains auteurs dans l'Essterel.

- Cistus poputitolius. Ça et là en Esspagne et, en France, dans les Pyrénées orientales et dans les Corbières.

- Cistus hirsutus. Fréquent dans tout le centre et l'ouest de 1'Espagne. N'ex iste en France que dans une station isolée du Finistère à Poularvelin.

- Cistus heterophyllus. Fréquent en Espagne centrale, occidentale et méridionale. N'existe pas en France.

Nous nous trouvons donc en présence d'un certain nombre d'espèces qui peuvent être considérées comme caractéristiques et que nous avons effectivement mises en évidence dans les miels espagnols: Citrus hirsutus ( $\left.{ }^{(}\right)$, Cistus heterophyllus et Cistus mumbyi.

Du point de vue morphologique, les différents Cistes sont assez difficiles à différencier. Les grains de pollens sont tous tricolporés et ne se distinguent entre eux que par la taille, 1'épaisseur et l'ornementation de l'exine qui passe du réticulum à larges mailles an granulum fin. La forme du sillon et des pores est caractéristique mais souvent fort pert visible sur les grains de pollen non fossilisés. Les grains de pollen

(1) La station bretonne ne joue aucun rôle; de toute façon un miel breton ne saurait être confondu avec un miel espagnol. 
des Cistacées français ont été décrits par M. T. JEAx et A. Poxs, (I962-I063). Nous décrirons donc seulement les espèces caractéristiques des miels espagnols

Cistus hirsutus. Axe polaire 47 à $55 \mu$. Diamètre équatorial 45 à $50 \mu$. Exine de 3,75 à $5 \mu$ d'épaisseur. Réticulum fin, luminae de I $\mu$ de diamètre. Sillon long et mince, pore légèrement ovale transversal de $9 u$ de diamètre.

Cistus heterophyllus. Axe polaire 43 à $48 \mu$. Diamètre équatorial 38 à $47 \mu$. Exine $3 \mu$ d'épaisseur. Reticulum fin, diamètre des luminae $I \mu$. Sillon long, de $I, 5 \mu$ de largeut. Largeur du sillon uniforme. Pore presque rond $(\delta \times 7 \mu)$. Pollen assez globulaire.

Cistus mumbyi. Axe polaire $50 \mu$, diamètre équatorial 45 à $50 \mu$. Exine de 3 à $4 \mu$ d'épaisseur. Reticulum fin mais visible (1uminae I $\mu$ ). Sillon long et mince. Pore ovale et transversal peu visible.

Cistus heterophyllus est courant dans les miels d'Espagne (tabl. I). Inconnu en France, il permet de distinguer d'une façon sûre les miels espagnols. Cistus mumbyi est également absent de France mais nous ne 1'avons trouvé que rarement dans les miels espagnols. Il est surtout caractéristique de 1'Afrique du Nord et ne se trouve que dans les miels espagnols d'origine nettement méridionale, accompagnant le plus souvent 1'Oranger, l'Olivier et l'Eucalyptus. En définitive, on ne le trouve que dans les spectres inconnus dans leur ensemble en France et s'il constitue 1ne caractéristique il n'intervient que dans une faible part dans le diagnostic final. Cistus hirsutus est plus fréquent que $C$. mumbyi mais sa répartition est cependant moins large que celle de Cistus heterophyllus. Si ce dernier constitue la Cistacée la plus typique des miels espagnols, $C$. hirsutus aidera souvent pour porter le diagnostic final.

Labiées. - Les I abiées interviennent largement dans le spectre pollinique des miels espagnols. Nous nous sommes limités au genre Lavandula et nous avons dressé le tableau suivant des aires de répartition géographique.

Genre Lavandula. Lavandula vera et L. latifolia se rencontrent en France et en Espagne. Le lavandin, cultivé en France sur de grandes surfaces, peut se rencontrer à l'état sauvage en Espagne.

Lavandula stoechas. Plante courante en Espagne, elle se rencontre aussi en France surtout dans 1'Esterel et dans les Pyrénées orientales.

Lavandula dentata. Cette plante est bien caractéristique de la moitié sud de la péninsule ibérique (régions australes et orientales). Elle n'existe pas en France si ce n'est dans quelques jardins autour de Nice (BARBIRR, I963).

Nous avons vu que Lavandula vera est souvent présente, parfois dominante dans les spectres espagnols. Lavandula latifolia est nettement plus rare mais peut être rencontrée cependant à un pourcentage élevé (miel 580 ). Il n'en reste pas moins vrai que Lavandula stoechas et Lavandula dentata sont rencontrées d'une façon beaucoup plus générale, mais également à l'état moins dominant. I,es miels purs de Lavandula stoechas restent plus rares que ceux de Lavandula vera. Cette dernière a été rencontrée dans I I des miels ici étudiés, $L$. stoechas dans 2 I et $L$. dentata dans I I également. Lavandula stoechas ne peut être considérée comme une espèce type des spectres espagnols. Beaucoup de spectres français la présentent. Par contre, L. dentata n'a jamais été décelée dans un spectre français. Elle ne se trouve sur le territoire français que très rarement, seulement à l'état isolé dans quelques jardins del'agglomération niçoise.

Lavandula dentata constitue donc une excellente caractéristique, d'autant plus que son pollen se distingue nettement de celui de $L$. stoechas dont il est cependant voisin. 


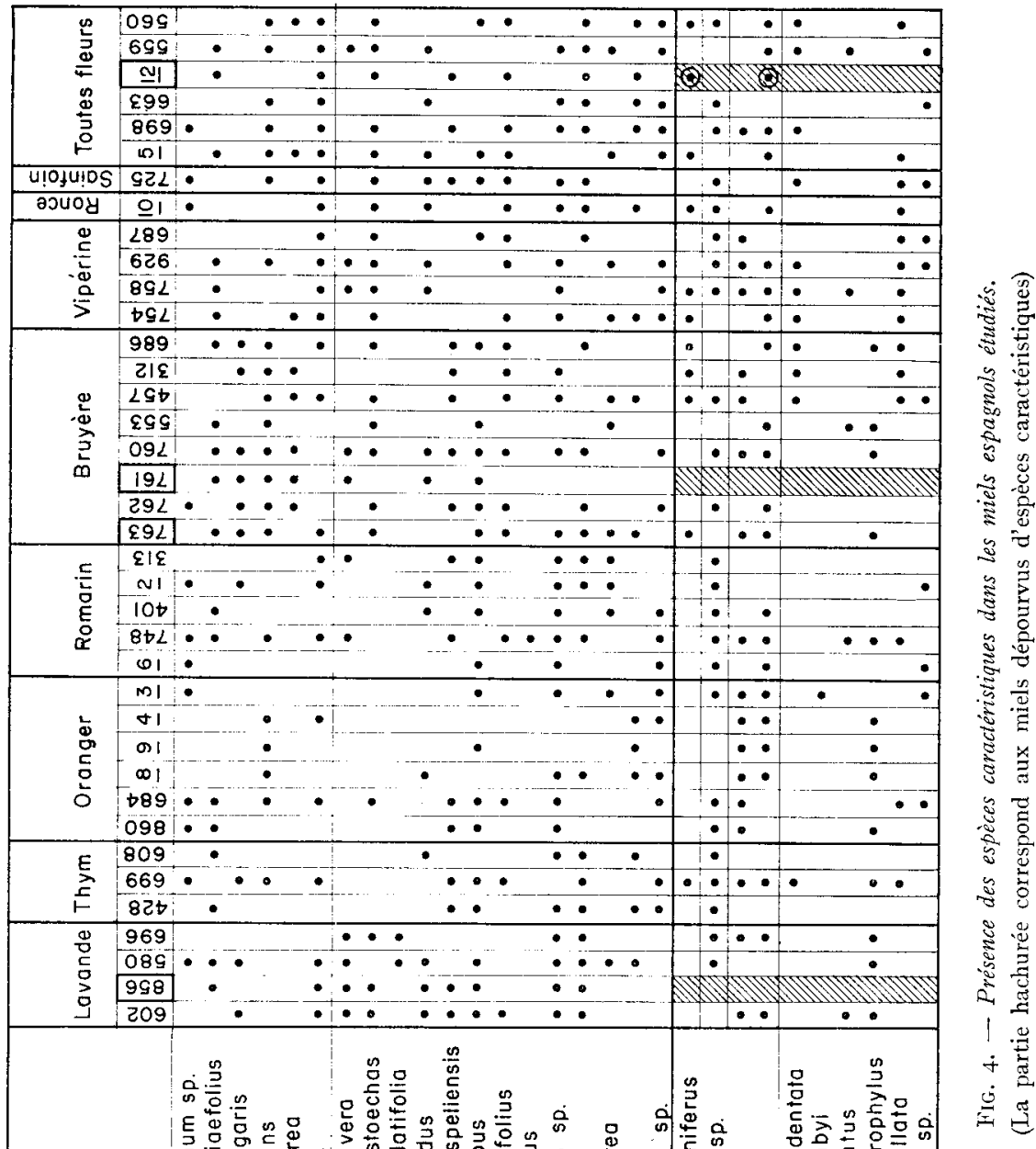
施

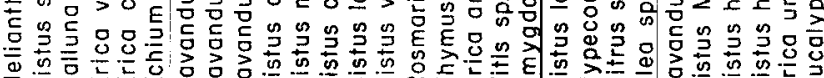

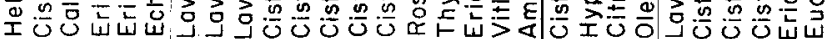

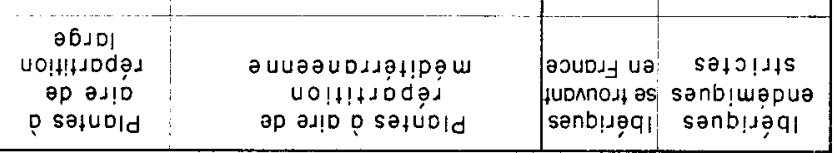


Lavandula dentata a été décrite par BARBIER (I963). Pollen hexacolpé, axe polaire 27 à $29 \mu$. Diamètre équatorial 33 à $3^{8} \%$. Six colpus bien délimités (largeur des colpus $7 \mu)$. Exine assez épaisse $(2 \quad \ell)$ et finement ornementée. Coloration du cytoplasme jatne.

Les autres Lavandes ont été souvent décrites et sont facilement déterminées.

Bien entendu, les genres Citrus et Eucalyptus peuvent, eux aussi, être considérés, dans une certaine mesure comme des caractéristiques. Cependant, leur présence dans les miels français ne peut être totalement exclue et ils ne prennent toute leur valeur que dans la mesure oì le reste du spectre pollinique est en accord avec une origine espagnole. Par ailleurs, Citrus, aussi bien qu'Eucalyptus, interviennent dans trop de spectres polliniques de miels exoíiques pour qu'on puisse porter le diagnostic de miel d'Espagne sur leur simple présence.

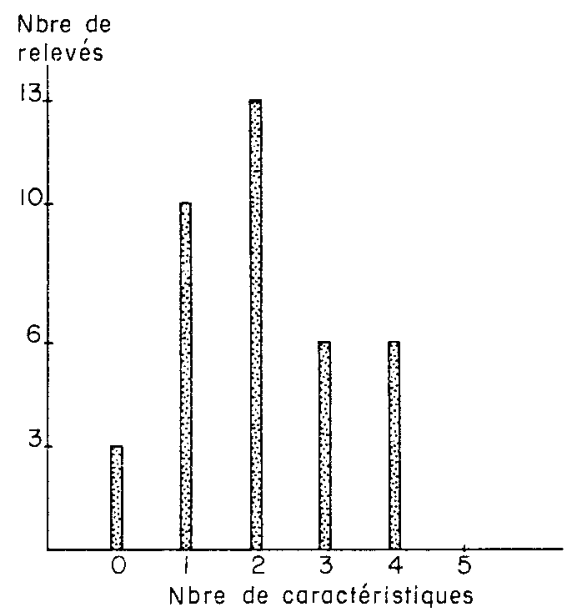

FIG. 4 bis - Diagramme en batons: nombre d'espèces caraciéristiques des trente-luit spectres espagnols

En résumé, nous pouvons donc considérer comme différenciant sans ambiguité les miels espagnols des miels français les espèces végétales suivantes:

Hypecoum sp., Lavandula dentata, Cisius heterophyllus, Cistus hirsutus, Erica umbellata et Cistus mumbyi. De plus Citrus sp. et Eucalyptus sp. n'existent pas dans le spectre des miels français dans des pourcentages dépassant à peine quelques unités.

En considêrant cette liste (à l'exclusion de Citrus), nous avons dénombré le nombre d'espèces caractéristiques par échantillon et nous avons construit le diagramme ci-dessus. Nous constatons que seuls 3 spectres polliniques sont dépourvus d'espèces caractéristiques (voir fig. 4 et + bis). I1 s'agit des miels 856, 76I et I2. Remarquons cependant que le miel de Lavande 856 n'est absolument pas conforme à l'idée générale que l'on se fait des miels de Lavande français. Le diagnostic reste alors facile à faire. Si l'origine des miels I 2 et $76 \mathrm{I}$ n'avait pas été connue comme espagnole, le diagnostic se serait limité sans doute à une forte présomption.

Annales de l'Abeille. - I964. 


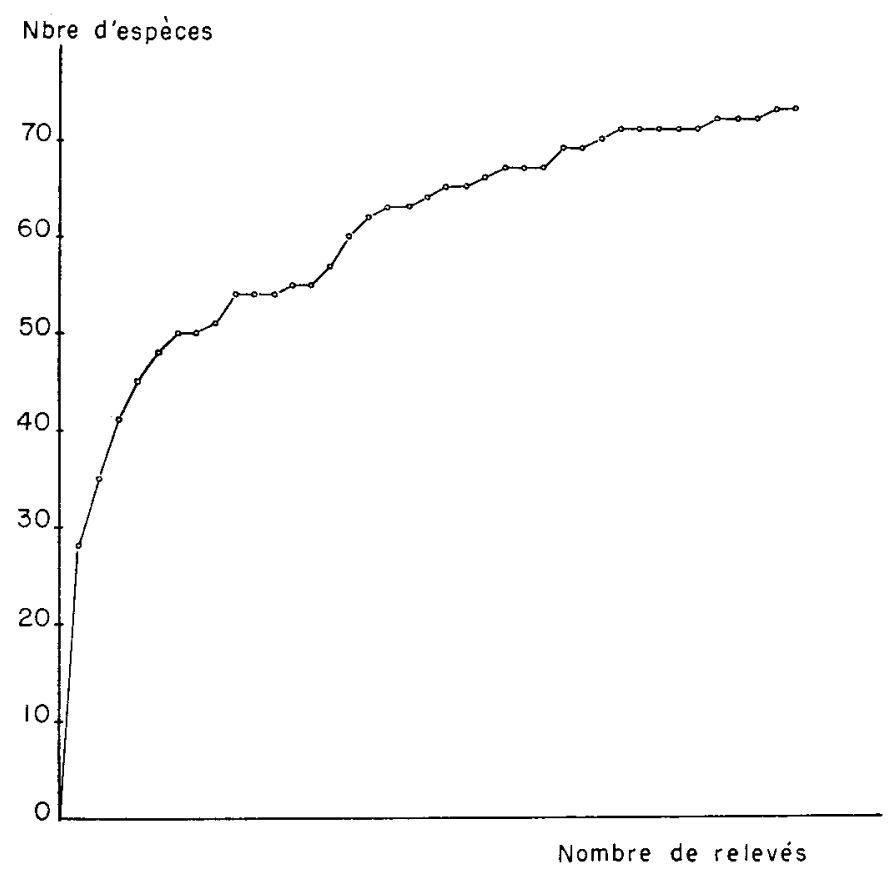

FIG. 5. - Courbe du type de celle de Gunochet (1955) obtenue à-partir de trente-huit relevés

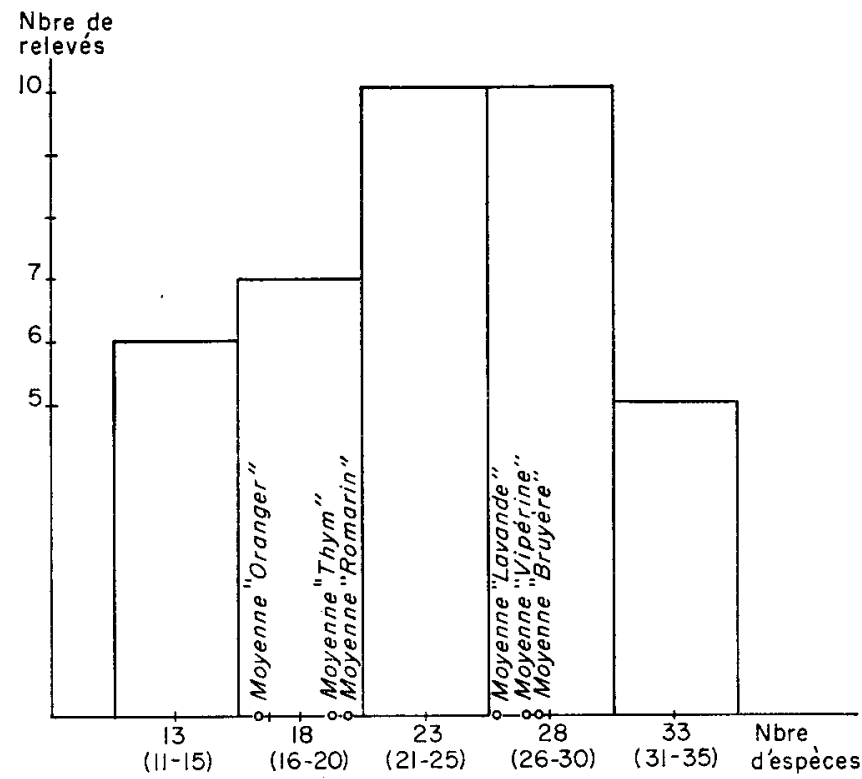

FIG. 6. - Histogramme de fréquence

(verticalement nombre de relevés, horizontalement nombre d'espèces) 


\section{$2^{\circ}$ Richesse en espèces des miels d'Espagne}

Le nombre total d'espèces rencontrées dans les $3^{8}$ spectres est assez important: 73 (fig. 5). Il semble suffisant pour se faire une bonne idée des miels espagnols. En effet si nous cumulons les nombres d'espèces obtenures dans chaque analyse nous obtenons une courbe du type de celle de Gunochet (I955). Nous observons la formation d'un début de palier. Nous pouvons donc en conclure que toutes les espèces couramment rencontrées dans les spectres polliniques des miels espagnols ont été observées dans le cadre de ce travail. L'utilisation de ce type de courbe n'est évidemment que très approximative ; certains grains de pollens ne sont, en effet, déterminés qu'au niveau du genre (auquel peut correspondre plusieurs espèces) ou même sont rapportés à un type plus général (arbres fruitiers, type Ulex-Sarothamnus). Nous avons déjà remarqué que le spectre pollinique des miels espagnols est typiquement riche en espèces. La moyenne du nombre d'espèces pour les 38 spectres présentés est de 23,2 et la trentaine d'espèces est très généralement dépassée surtout pour les miels de Bruyère, de Vipérine et les miels dits " toutes fleurs" (voir fig. 6). Par contre, les miels d'Oranger, et de 'Thym sont typiquement pauvres en espèces. Pour ce qui est des échantillons étudiés, nous constatons que plus de la moitié correspond à un spectre comportant de 2 I à 30 espèces. Seul le tiers environ est représenté par des spectres à moins de 20 espèces, et cette partie n'est pratiquement composée que de miels d'Oranger, de Romarin et quelques miels de Thym.

\section{CONCLUSION}

La recherche des miels espagnols vendus sous de fausses appellations françaises se trouve facilitée par la connaissance des espèces citées ci-dessus et $90 \mathrm{p}$. Ioo des fraudes devraient, ainsi, être décelées.

Cette proportion, quoique fort satisfaisante, doit être améliorée et pour y parvenir nous devons rechercher d'autres espèces caractéristiques courantes en Esspagne. En particulier, l'éventail des familles étudiées doit être élargi. Ainsi, les Composées présentent dans les spectres polliniques des miels espagnols des formes typiques encore inconnues. Les Papilionacées gagneraient également à être étudiées d'une façon approfondie. Mais leur morphologie rend souvent difficile une détermination fine des grains de pollen.

Les spectres proposés dans cette étude sont encore, dans le détail, incomplets, mais les connaissances acquises permettent de parvenir dans une grande mesure au but que nous nous étions fixés. La reconnaissance de quelques grains de pollen au niveau de l'espèce est en définitive plus rentable que celle d'un plus grand nombre au niveau du genre ; l'utilisation de spectres polliniques légèrement incomplets est plus déterminante que celle de spectres imprécis.

C'est alors le choix des familles qui seront particulièrement étudiées qui devient important. L'approfondissement de nos connaissances ne doit pas se faire d'une façon dispersée mais sur des points bien précis. Il existe pour chaque problème une ou plusieurs " espèces-clé " que nous devons rechercher dans des familles présentes d'une façon suffisamment générale. 


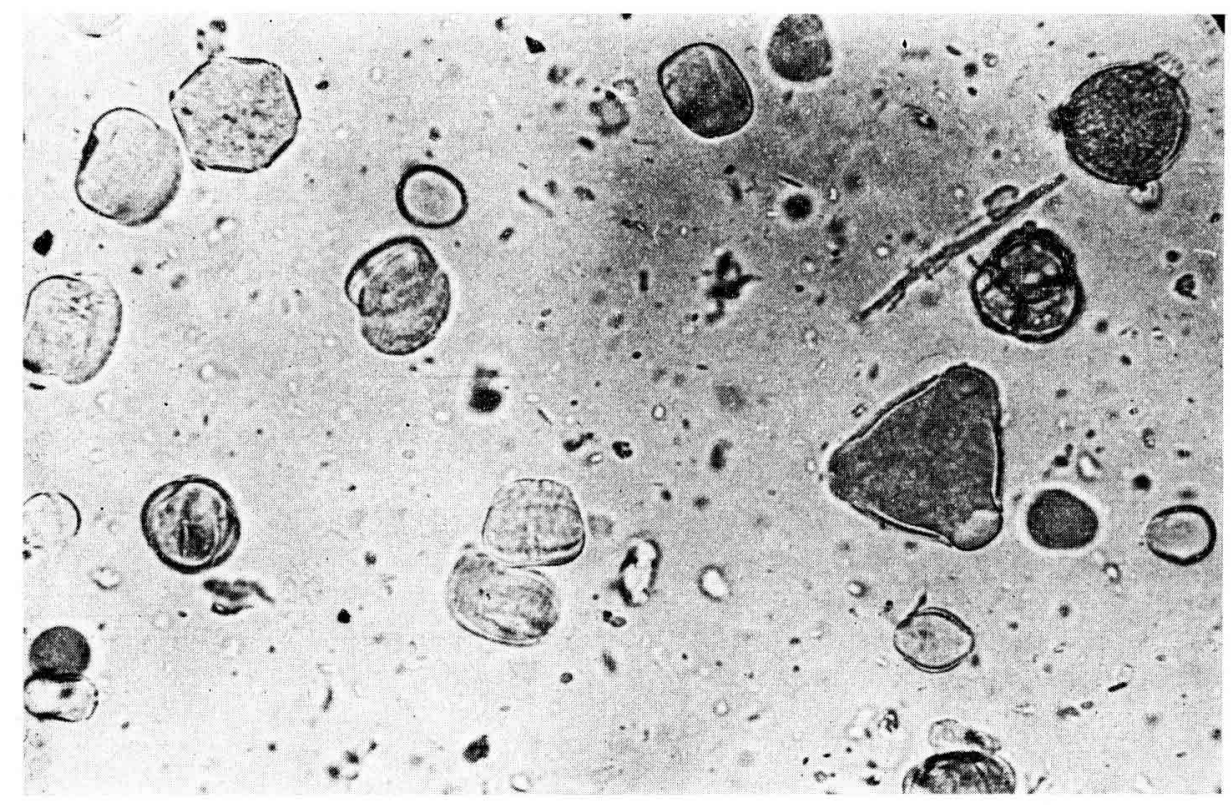

(a)

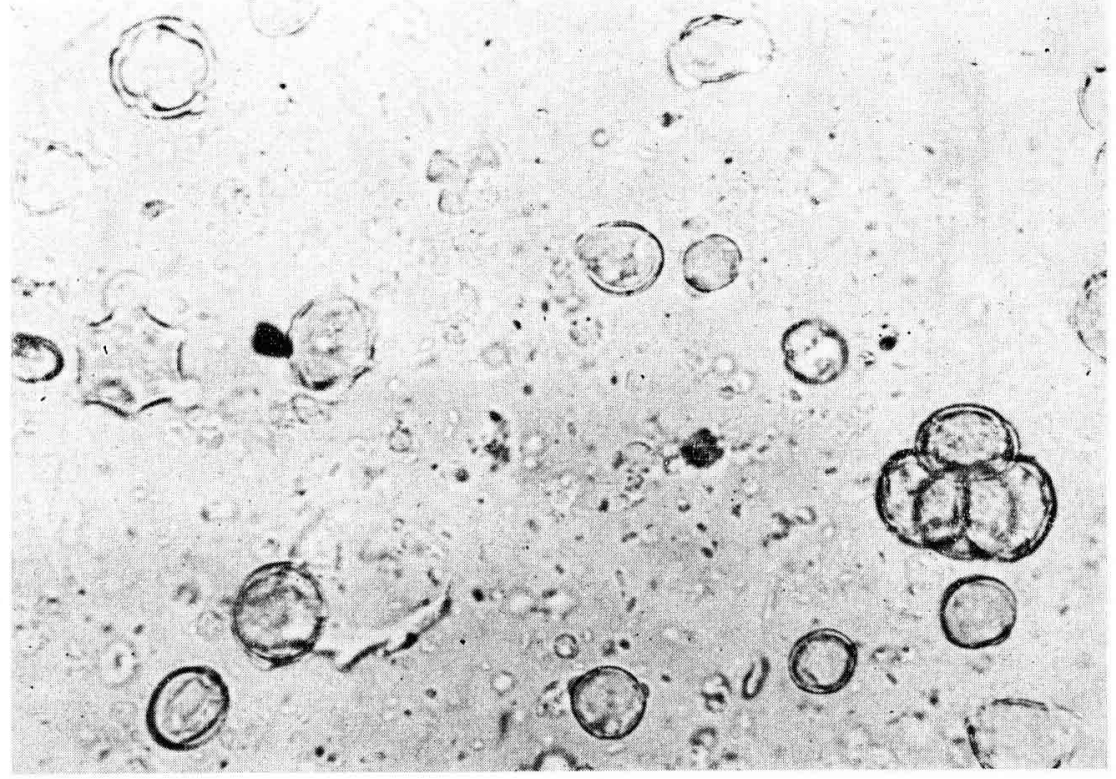

(b)

Eléments du spectre pollinique de quelques miels espagnols caractéristiques

P'LANCHE I a) Onobrychis sativa, Amygdalus sp., Hypecoum sp., (en bas à droite), Echium sp., Thymus sp., Erica arborea,

b) Citrus sp., Lavandula stoechas, Rubus ps., Vitis sp., Erica umbellata 


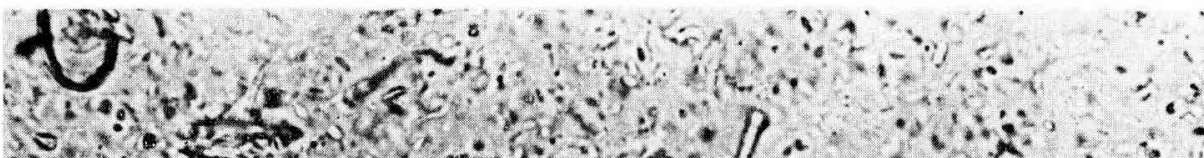

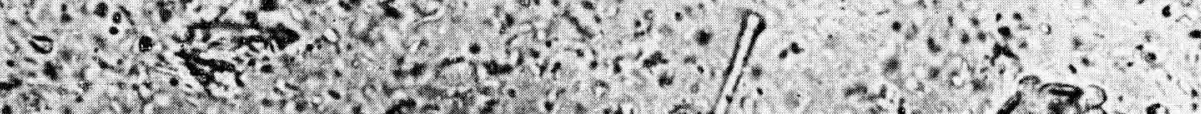





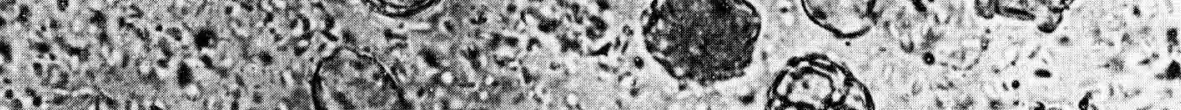

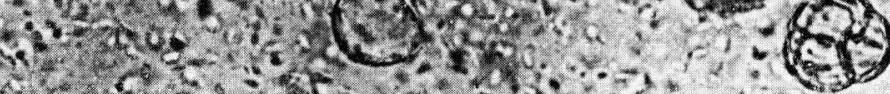

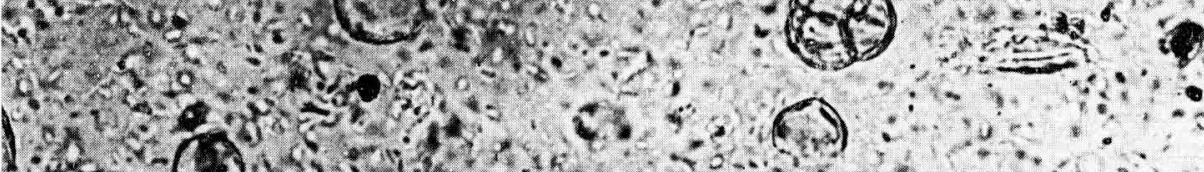

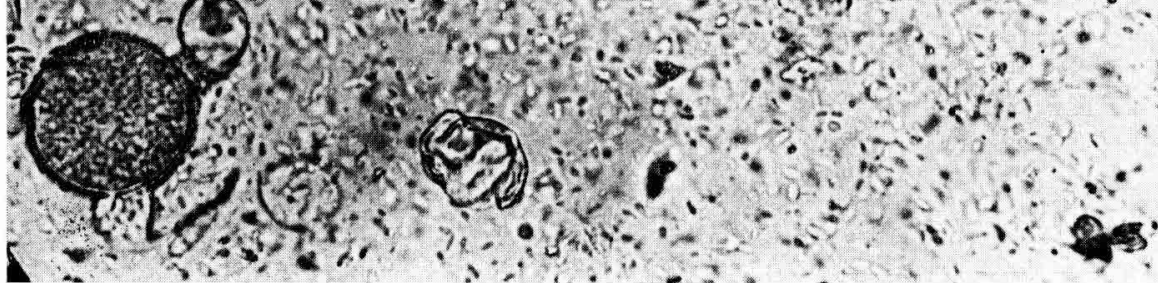

(a)



(b)

PLANChe 2

a) De droite ì garcche

Lavandula vera, Rubus sp., Erica arborea, Lavandula sloechas, Vitis sp., Rubus sp., Cistus hirsutus.

b) De gauche à droite

Cistus laurijolius et Vilis, Citrus sp., Helianthemun s!.., Cistus mumbyi, Rubus sp., Erica vagans, Olea eutropa, Vitis sp. 




(a)



(b)

Planche 3

a) de gauche à droite: Erica arborea et Cistaceae, Lazandula dentata, Erica umbellata et Cistus heterophyllus, Eucalyptus sp.

b) Dominance Erica vagans. Calluna vulgaris, Hypecoum sp., Onobrychis sativa, 


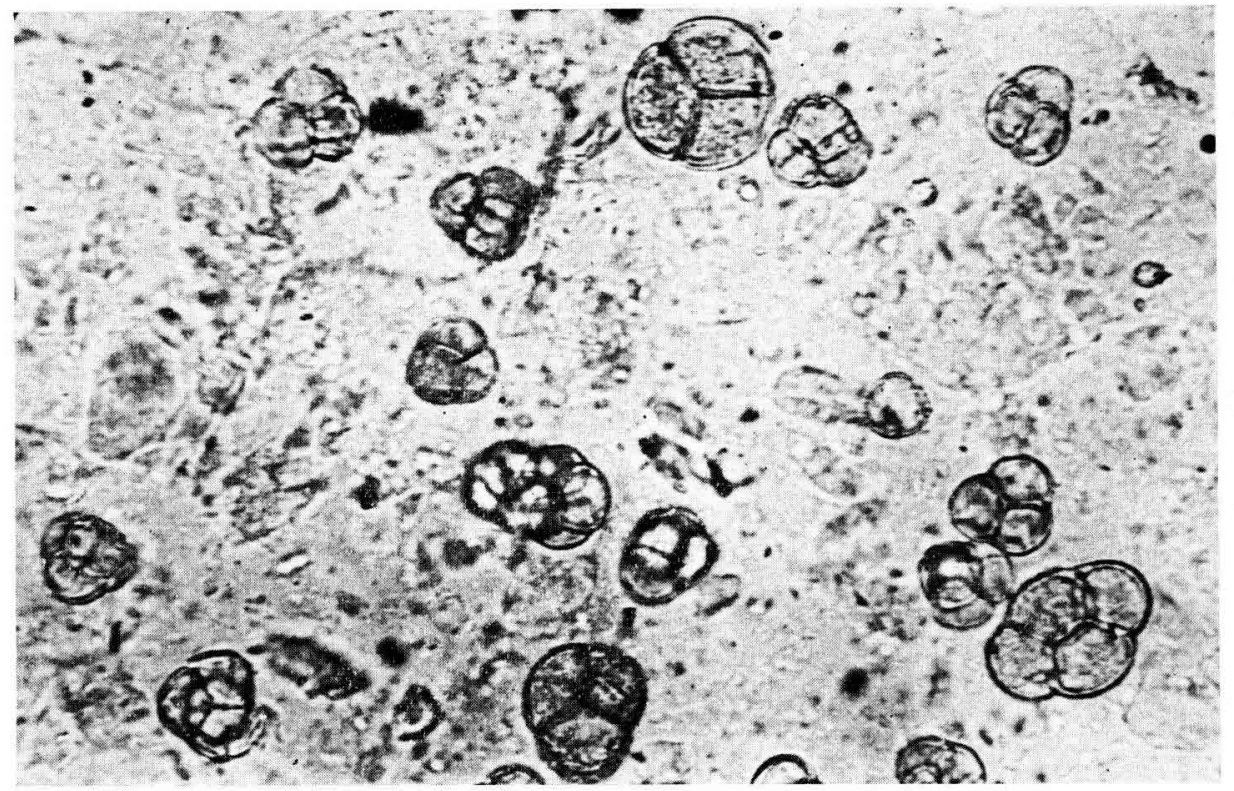

(a)

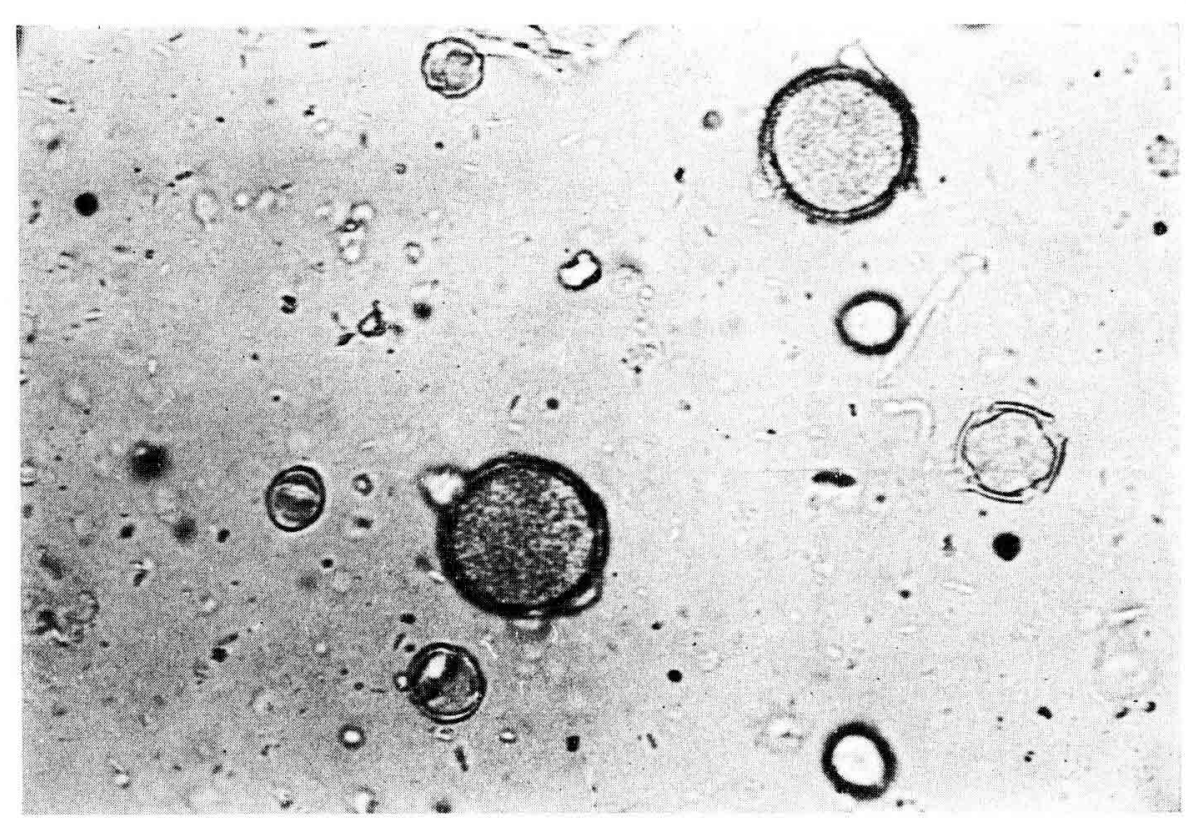

(b)

PLAXCHE 4

a) Association Erica zagans, Calluna aulgaris et Erica cinerea. Salix sp.

b) Cistus hirsutus et Vitis, Citrus sp. 


\title{
RÉSUMÉ
}

\author{
ḰTUDE IOU SPECTRL POLLINIQLE DE OUELGUES MIELS IESAGNOLS
}

Trente-huit miels espagnols ont été étudiés. Les préparations pollini fues ont été obtenues velon la méthode de MaUrizio-Jocvisux. Cés spectres pollinifues ont été établis et ont permis de repérer des grains de pollens correspondant à des plant es non retrouvées dans les spectres polliniques français.

Tne étude approfondie de l'aire de répartition de certaines Papaveracées, Fricacées, Cistacées ct Labiées a été faite. (On a ainsi déterminé que Hypecotum sp. souvent trouvé dans les miels uspagnols en est bien caractéristique. Parmi les Éricacées, Errica umbellata ne se retrouve que dans les spectres espagnols. De plus, des associations avant pour base Lirica vagans et Callune sont bien caractéristiques des miels de l'Espagne occidentale èt différent largenent des miels de Bruyère du Sud-Ouest de la France.

Les ('intacées sont très variées et présentes en grande quantité dans les spectres des miels espagnols. C'istus hirsutus, Cistus heterophyllus et Cistus mumby'i rencontrés dans les échantillons espagnols ne peuvent être butinés en France.

Laqandula dentala est la seule Iavande pouvant différencier les deux types de spectres étudiés : elle ne se rencontre pas en France.

Enfin, les spectres des miels d'Espagne sont souvent caractérisés par leur complexité et le plus grand nombre d'espèces $y$ intervenant.

\section{SUMMARY}

STUIY OF THE POLLEN SPECTRLIM OF CERTAIN SPANISH HONEYS

Thirty-eight Spanish honeys have been studied. The pollen preparations were obtained by the Maurizio-Louveatx method. These pollen spectra have been obtained and have revealed pollen grains from plants which are not to be found in lirench pollen spectra.

An exhaustive study of the area of distribution of certain Papaveraceae, Ericaceae, Cistaceae and Labiatae was conducted, from which it was determined that Hypetoum sp., frequently found in Spanish honeys, is highly characteristic. Furthermore. associations based on lirica vagans and Calluna are very characteristic of western spain and differ considerably from the heather honeys of south-western France.

The Cistaceae occur many varieties and in large quantities in the spectra of Spanish honeys. Cistus hirsutus, Cistus heterophyllus and Cistus. Humbyi encountered in the Spanish samples cannot be exploited in France.

Lazandula dentata is the only lavender which can dist inguish the two types of spectrum studied: it is not found in France.

\section{RÉFÉRENCES IBIBLIOGRAPHIQUES}

Barbler E., 1963. Ies lavandes et lapiculture dans le sud-ent de la France. Amn. Abeille, 6 (2), 85-159.

Bonvier (i., I934. Flore complete illustrée en rouleurs de Trance, Suisse of Belgique (comprenant la plupart des plantes d'Europe), i 2 vol., lE. (Orlhat, l'aris.

Cabaldero A., 1970. Flora analitica de Espana, Sociedad Anmima Espruola de Traductores y Autores, Madrid.

Coste IH., 1937. Flore descriptive et illustrée de la France, de lia Corse et des contrées limitrophes... avec une introduction sur la Hore et la végétation de lit France..., 3 vol., librairie des Sciences et des Arts, Paris.

ERDTMan (;., I952. Pollen morphology and plant taxonony. Angiosperms (an Introduction to Palynology I), Almquist et Wiksell :Stockholm. 
Gunocher M., 1955. Logique et dynamique du peuplement végétal, Masson d Cie, l'aris.

Jean M. T., Pons A., rgoz. Tine clef de déternination palynologrique pour les (istacées de la lilore de France. Naluratia Monspeliensia. Série TBotanique, fisc. $1+4,8 ;-02$.

JeAn M. T., PoNs A., iof.3. Contribution a l'etude palyologique des Cistacés de la Flore de France. Ann. Sci. Nal. Bolan. Végét., $12^{\mathrm{e}}$ séric, 4 (1), 150-1;0.

Martixs D'Aite J. A., 1951. Analyse polinica de algumas anostrats de mel. Publ. Inst. Botan. "Ai Gonsalo Sampaio ", $2^{\mathrm{e}}$ série, $n^{\prime \prime} 7$.



Maurizo A., Jouvenux J., т1)63. Méthodes d'analyse pollinique des nuicls. Ann. Abeille, 6 (1), $75-76$.

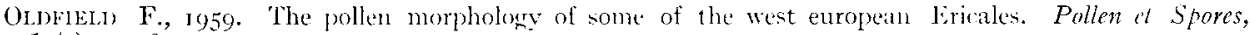
1 (I), $19-48$.

Vinitez F., r950 a. El polen de las unieles de Gialicia. Anales de Edajologin y Fiviologia regelal, 10, $79^{-100 .}$

VIEITEz li., 1950 b. Palynological olservations on some spanish honeys. Bull. of the Torey Botanical Club, 79 (6), $495-502$.

Wulkom M., IAANik J., i 870 . Prodonus Floral Hispanicae seu Synopsis methodica omnimm plantarum in Ilispania. 3 vol., E Koch, Stuttratr. 